Urologe $2021 \cdot 60: 796-802$

https://doi.org/10.1007/s00120-021-01566-w Online publiziert: 20. Mai 2021

(c) Springer Medizin Verlag GmbH, ein Teil von Springer Nature 2021

\section{Es ist nicht nur die Erkenntnis aus der aktuellen COVID-19-Pandemie; sondern seit Jahren spürbar: Der Pflegenotstand nimmt zu. In der aktuellen Situation wird nur noch einmal überdeutlich, wie groß der Notstand ist und wie angewiesen wir auf eine bedarfsgerechte Pflegepersonalausstattung sind, damit die Versorgung der Patienten abgesichert werden kann.}

In 2 aktuellen Positionspapieren „Mehr PflegeKRAFT 2.0 - Pflege ist mehr als systemrelevant" des Pflegebevollmächtigten der Bundesregierung, Staatssekretär Andreas Westerfellhaus [1], und auch in der 4. Ad-hoc-Stellungnahme „CoronavirusPandemie: Medizinische Versorgung und patientennahe Forschung in einem adaptiven Gesundheitssystems "der Leopoldina [2] wird neben anderen Maßnahmen auch zur Lösung des Personalmangels die Einführung einer einheitlichen Personalbemessung gefordert. Warum wird das als Lösung gesehen? Womit kann die Personalbemessung erfolgen? Wie stehen dazu die Pflegepersonaluntergrenzen?

Die aktuelle angespannte Personalsituation in der Pflege ist aber nicht die erste Situation dieser Art. In den 1980erJahren gab es ebenfalls einen Pflegenotstand in den deutschen Krankenhäusern. Nach massiven Protesten hunderttausender Pflegefachpersonen lenkte der damalige Gesundheitsminister Horst Seehofer ein und brachte ein Personalbemessungsverfahren auf den Weg, die Pfle-

Dieser Beitrag wurde zuerst publiziert in Jahn $\mathrm{P}$ (2020) Untergrenzen oder Personalbemessung in der Pflege. Onkologe 26:1040-1046. https:// doi.org/10.1007/s00761-020-00838-y

\title{
P. Jahn
}

Department für Innere Medizin, AG Versorgungsforschung, Pflege im Krankenhaus, Universitätsmedizin Halle (Saale), Halle (Saale), Deutschland

\section{Untergrenzen oder Personalbemessung in der Pflege}

gepersonalregelung (PPR). Diese wurde 1993-1995 verpflichtend eingeführt und bereits 1996 wieder ausgesetzt, weil sie mit 52.000 Stellen deutlich mehr Bedarf ergab, als erwartet worden war [3]. Die PPR wurde jedoch auch nach Aussetzung in vielen Einrichtungen weiter zur internen Personalsteuerung verwendet und ist u.a. Grundlage für die Kalkulation der Pflegepersonalkosten und weiterer Kosten in den Diagnosis Related Groups (DRG) oder Fallpauschalen [3, 4].

Mitte der 2000er-Jahre läutete die Einführung der DRG den Paradigmenwechsel in der Krankenhausfinanzierung ein. Die marktwirtschaftliche Orientierung führte im Zusammenwirken mit einigen anderen Faktoren (z. B. unzureichende Investitionskostenfinanzierung der Bundesländer) v. a. zu einem Abbau von Pflegepersonal [5]. Das Personalbudget für die Pflege musste herhalten, um die finanziellen Löcher zu stopfen und ausgeglichene Zahlen in den Bilanzen zu erreichen.

Mit dem Pflegepersonal-Stärkungsgesetz (PpSG) wurden unter Gesundheitsminister Spahn nun über die Ausgliederung des Pflegepersonalbudgets und die Einführung von Pflegepersonaluntergrenzen (PpUG) Maßnahmen zur Gegensteuerung beschlossen. Die Ausgliederung der Pflegepersonalkosten - und die damit vollzogene Rückkehr zur vollständigen Deckung der Kosten für das Pflegepersonal - kann, wenn dies um eine einheitliche und bedarfsgerechte Personalbemessung ergänzt wird, tatsächlich einen Ausbau der Pflegestellen bewirken. Die Umsetzung der vom Bundesministerium für Gesundheit (BMG) festgelegten PpUG wird von vielen Verbänden eher als problematisch eingeschätzt, auch wird sie durch ein Personalbemessungsverfahren überflüssig.

\section{Kritische Betrachtung der Pflegepersonaluntergrenzen}

Die initiale Entscheidung zu pflegesensitiven Bereichen und den dazugehörigen PpUG ist aus inhaltlicher und methodischer Sicht zu kritisieren. Erhoben wurden diese auf der Grundlage von Daten der Qualitätsberichte über die Personalausstattung in den einzelnen Fachabteilungen deutscher Kliniken die auf eigenen Angaben der Kliniken beruhten und mit den Abrechnungsdaten (Daten nach $\$ 21$ Krankenhausentgeltgesetz, KHEntgG) zusammengeführt wurden - [5]. Die Zusammenführung ist vor dem Hintergrund der zwischen den Einrichtungen sehr unterschiedlichen Darstellung der Personalzahlen pro Fachabteilung sehr fraglich. AuBerdem wurden die „pflegesensitiven Ergebnisindikatoren" (PSEI) entwickelt, um fachabteilungsunabhängig den $\mathrm{Zu}$ sammenhang zwischen Pflegepersonalausstattung und unerwünschten Patientenereignissen zu ermöglichen [6,7]. In der den PpUG zugrunde liegenden Expertise wurde aber unterstellt, dass für bestimmte Abteilungen ein besonderer Zusammenhang besteht und diese damit besonders pflegesensitiv sind [5].

Außerdem wurde die Grenze willkürlich bei den 10-25\% Kliniken mit der schlechtesten Ausstattung gezogen. Dieses Niveau für die Personalbesetzung wurde als sichere Versorgung definiert. Eine fachliche Begründung dafür oder eine Bewertung der damit erreichten Pflegequalität sind nicht bekannt. Bedenklich ist dies auch vor dem Hinter- 


\begin{tabular}{lllll}
\hline & Früh/Spät & $\begin{array}{l}\text { Anteil } \\
\text { Pflegehilfe }\end{array}$ & Nacht & $\begin{array}{l}\text { Anteil } \\
\text { Pflegehilfe }\end{array}$ \\
\hline Intensiv* & $2,5: 1$ & $8 \%$ & $3,5: 1$ & $8 \%$ \\
\hline Unfallchirurgie & $10: 1$ & $10 \%$ & $20: 1$ & $15 \%$ \\
\hline Geriatrie & $10: 1$ & $20 \%$ & $20: 1$ & $40 \%$ \\
\hline Kardiologie & $12: 1$ & $10 \%$ & $24: 1$ & $15 \%$ \\
\hline Herzchirurgie & $7: 1$ & $5 \%$ & $15: 1$ & $0 \%$ \\
\hline Neurologie & $10: 1$ & $10 \%$ & $20: 1$ & $8 \%$ \\
\hline Stroke Unit & $3: 1$ & $0 \%$ & $5: 1$ & $0 \%$ \\
\hline Neurol. Frühreha. & $5: 1$ & $10 \%$ & $12: 1$ & $8 \%$ \\
\hline * Ab 01.01.2021 im Intensivpflegebereich TD 1:2 und ND 1:3 & &
\end{tabular}

Abb. 1 \ Vorgaben zur Pflegepersonalausstattung gemäß Pflegepersonaluntergrenzen-Verordnung (PpUGV; §137i Fünftes Buch Sozialgesetzbuch, SGB V). TD Tagdienst, ND Nachtdienst

grund, dass die Ausstattung mit Pflegepersonal im europäischen Vergleich in Deutschland besonders schlecht ist. Diese liegt für die wichtigsten Belastungsindikatoren Vollkräfte (VK) pro 1000 Fälle oder VK pro 1000 Betten weit hinter dem Durchschnitt der Organisation für wirtschaftliche Zusammenarbeit und Entwicklung (OECD) [8, 9]. In Zeiten knapper Personalressourcen in der Pflege im Krankenhaus wird hier für die Mehrheit der Kliniken eine Grenze definiert, die unterhalb der bisherigen Besetzung liegt.

\section{》) Hier wird für die PpUG eine Grenze definiert, die meist unterhalb der bisherigen Besetzung liegt}

Außerdem ist die Bildung von PpUG an die Fachabteilung gebunden, die onkologische Pflege lässt sich aber - bis auf die Hämatologie und Strahlentherapie - nicht über die spezifische Fachabteilung abbilden, wie beispielsweise für die Neurologie. Die onkologischen Patienten werden auf vielen unterschiedlichen Abteilungen versorgt, z. B. Gynäkologie, Urologie oder Dermatologie.

Nachdem zunächst nur für 4 Fachbereiche PpUG festgelegt wurden, wurden diese 2019 mit Gültigkeit ab 01.01.2020 um weitere 4 Bereiche erweitert (• Abb. 1) Dabei kommt es zu unerwünschten $\mathrm{Ne}$ beneffekten: Der Zuschnitt von Stationen und Abteilungen wurde angepasst, um möglichst wenig von der verpflichtenden Umsetzung der PpUG betroffen zu sein, Pflegepersonal wird in die geregelten Bereiche versetzt - zulasten der nichtgeregelten Bereiche, Patienten werden möglichst in nichtbetroffene Bereiche verlegt. Und immer mehr Pflegedirektor*innen werden mit Forderungen der Geschäftsleitung konfrontiert, den Personalschlüssel in Richtung Untergrenze abzusenken.

Hinzu kommt, dass bei den Untergrenzen eine Fachkraftquote formuliert ist. Auch diese Festlegung erfolgte ohne jegliche fachliche Rechtfertigung. Verbesserungen in begrenztem Umfang werden also höchstens die $25 \%$ der am schlechtesten mit Pflegepersonal ausgestatteten Krankenhäuser haben, soweit sie nicht die Anforderungen geschickt umgehen. Die Nebenwirkungen der Therapie sind also möglicherweise schlimmer als die Grundproblematik. In einer aktuellen Umfrage des Deutschen Berufsverbands für Krankenpflege (DBfK) zeigen sich auch 2/3 der Pflegefachpersonen unzufrieden mit den Effekten der PpUG [10]. Zudem wurden bereits zum 4. März - im Zusammenhang mit den Maßnahmen zur COVID-19-Pandemie die PpUG durch das BMG befristet ausgesetzt. Es stellt sich die Frage, ob und wie die PpUG überhaupt nochmal zurückkommen und wie eine Lösung aussehen kann, die einen Aufbau des Pflegepersonals im Krankenhaus auf ein international anschlussfähiges Niveau fördern kann.

Neben den Maßnahmen des PpUG wird seit $2020 \mathrm{im}$ Zuge des PpSG das Pflegepersonalbudget unabhängig von den DRG krankenhausindividuell gebildet. Im Zusammenhang mit der Festschreibung des Budgets für die Finanzierung von Pflegestellen ist dies im Grundsatz ein richtiger Schritt vor dem Hintergrund der Erfahrungen der letzten 15 Jahre hinsichtlich der Entwicklung der Pflegepersonalsituation im Krankenhaus. Hier hat der Pflegekostenanteil innerhalb der DRG kontinuierlich abgenommen [8, 11].

Aktuell bleibt unklar, was bei der verbesserten Budgetsituation für das Pflegepersonal - das auf dem Markt derzeit nicht mehr zu finden ist - herauskommt. Positiv an diesem Ansatz ist, dass Mittel nicht mehr zweckentfremdet werden dürfen - für Arztstellen, Medizintechnik oder wo sonst noch das Geld fehlt. Sollte aber die Kalkulation nur den aktuellen defizitären und oft sicherheitsgefährdenden Stand abbilden, ist auch hier nichts gewonnen. Zudem ist problematisch, wie mit den unterschiedlichen Assistenzangeboten rund um die Pflege umgegangen werden soll. Zählt es zum Pflegebudget, wenn die Gesundheits- und Krankenpflegerin Aufgaben der Stationssekretärin übernimmt?

\section{》) Ein am Versorgungsbedarf ausgerichtetes Personal- bemessungsinstrument ist unverzichtbare Grundlage}

Auch hier ist ein am Versorgungsbedarf ausgerichtetes Personalbemessungsinstrument eine unverzichtbare Grundlage für die Berechnung des erforderlichen Budgets. Ein solches Instrument muss Auskunft darüber geben, welche Leistungen überhaupt von der Pflege erbracht werden sollen und welche pflegerische Qualifikation dafür jeweils erforderlich ist. 
Bemessung des Pflegepersonalbedarfs im Krankenhaus

\section{Entwicklung des Instruments PPR 2.0}

Im Rahmen der „Konzertierten Aktion Pflege" (KAP) der Bundesregierung haben sich der Deutsche Pflegerat e.V. (DPR), die Vereinte Dienstleistungsgewerkschaft (ver.di) und die Deutsche Krankenhausgesellschaft (DKG) verpflichtet, ein Instrument zur Bemessung des Personalbedarfs im Krankenhaus als Interimslösung $\mathrm{zu}$ erarbeiten und bis Ende 2019 dem BMG zur Bewertung und zur Entscheidung über eine weitergehende regulatorische Einbindung vorzulegen [12].

Ziel der 3 Organisationen ist es, die Arbeitsbedingungen in der Pflege zu verbessern und die Pflegepersonalbesetzung auf den Stationen der Krankenhäuser zu erhöhen, um die Attraktivität der Pflegeberufe zu steigern. Hierzu ist einerseits eine Pflegepersonalbedarfsbemessung notwendig, die verbindlich festlegt, welche Personalausstattung für die pflegerische Versorgung von Patienten angemessen ist, und andererseits sind Maßnahmen wie die Steigerung der Ausbildungskapazitäten, Rückgewinnung aus dem Pflegeberuf ausgeschiedener Pflegekräfte und Erhöhung der Stundenzahl von Teilzeitkräften erforderlich, um möglichst schnell eine Deckung des gemessenen Bedarfs zu erreichen.

》) Im Fokus standen Grund- und Fallwerte sowie Leistungsinhalte der allgemeinen und speziellen Pflege

Die nun als Interimslösung - entsprechend dem KAP-Auftrag - präsentierte PPR 2.0 ermittelt den Pflegepersonalbedarf eines Krankenhauses für die unmittelbare Patientenversorgung auf allen bettenführenden somatischen Stationen und definiert die notwendige Pflegepersonalausstattung für die Stationen des gesamten Krankenhauses [13]. Das Instrument der PPR 2.0 orientiert sich an den Standards einer qualitativ hochwertigen
Patientenversorgung und ist als Entwicklungskonzeption in $\mathbf{A b b} .2$ schematisch dargestellt.

Wie im Rahmen der KAP vereinbart, ist die Weiterentwicklung der PPR 2.0 hin zu einem wissenschaftlich fundierten, an den Versorgungsbedarfen ausgerichteten Pflegepersonalbedarfsbemessungsverfahren mittels eines Pflegepersonalbemessungsinstruments (PPBI) erforderlich. Es geht also um die parallele Entwicklung von 2 aufeinander aufbauenden Lösungsansätzen. Die PPR 2.0 als Interimslösung ermöglicht eine sofortige Einsatzfähigkeit und die wissenschaftliche Weiterentwicklung eine Langzeitlösung.

\section{Inhalt der PPR 2.0}

Im Detail stellt das neue Pflegepersonalbemessungsinstrument PPR 2.0 eine grundlegend überarbeitete und modernisierte PPR dar, die in ihrer ursprünglichen Form immer noch von vielen Krankenhäusern zur Kalkulation der vorhandenen Pflegepersonalkosten angewendet wird. Die Überarbeitung und Modernisierung der PPR zur PPR 2.0 erfolgte in einem mehrstufigen Prozess.

Im Fokus standen die Aktualisierung der Grund- und Fallwerte sowie die fachlich-inhaltliche Bewertung der Leistungsinhalte der allgemeinen und speziellen Pflege (A- und S-Bereich).

\section{- Grundwert:}

- insbesondere Berücksichtigung der erhöhten Anforderungen an Qualitätssicherung,

- Umsetzung neuer Pflegekonzepte,

- Pflichtweiterbildung

- Fallwert:

- Umsetzung von Expertenstandards und Leitlinien der Fachgesellschaften,

- Umsetzung des Entlassmanagements gem. Rahmenvertrag Entlassmanagement, \$39 1a Fünftes Buch Sozialgesetzbuch (SGB V)

- A-Bereich:

- u.a. Ablösung des Pflegekomplexmaßnahmen-Scores (PKMS) bei Erhalt der PPR-Stufe A4;

- Berücksichtigung aktivierender Pflegekonzepte;
- Anpassung der Zeitwerte für Pflegeplanung und -dokumentation an gestiegenen Umfang

- S-Bereich:

- u.a. Aktualisierung insbesondere der "pflegetechnischen Leistungen“ (delegierte ärztliche Tätigkeiten);

- Neukonstruktion der PPR-Stufe S4.

- Überprüfung der hinterlegten Zeitwerte,

- Überführung von dem bisherigen Zeitintervall von $14 \mathrm{~h}$ (6:00-20:00 Uhr)

- in ein 16 stündiges Zeitintervall (6:00-22:00 Uhr).

Bei der Überarbeitung konnte die eingesetzte Expertengruppe auf umfangreiche Vorarbeiten zurückgreifen. Meilensteine waren:

- Expertise zur Überarbeitung der PPR im Rahmen UAG (Unterarbeitsgruppe des Bundesministeriums für Gesundheit) Pflegestellenförderprogramm I (2008-2009)

- Entwicklung und Testung der Weiterentwicklung PKMS-E zu 2.0 und 2.1 (2011-2014)

- Entwicklung der PPR S4 und Einreichung in das Vorschlagsverfahren des Instituts für das Entgeltsystem im Krankenhaus (InEK, 2013-2016)

- Initiierung und Begleitung der Einführung der pflegerischen Leistungsabbildung über patientenbezogene Parameter wie Pflegegrad und Angaben, beispielsweise zur körperlichen Funktion, über Barthel-Index (U50/51) (2015-2018)

- Entwicklung des Pflegepersonalbemessungsinstruments (PPBI, seit 2018)

\section{Pretest zur Handhabbarkeit und Umsetzbarkeit}

Abschließend für die erste Entwicklungsphase wurde die PPR 2.0 einem Pretest zur Evaluation bezüglich Handhabbarkeit, Umsetzbarkeit und Verhältnis der Veränderungen zur PPR (alt) unterzogen. Der Pretest fand in einer Gelegenheitsstichprobe von 44 Einrichtungen im Zeitraum vom 04.11.2019 bis einschließlich 25.11.2019 statt. Die Evaluation wur- 


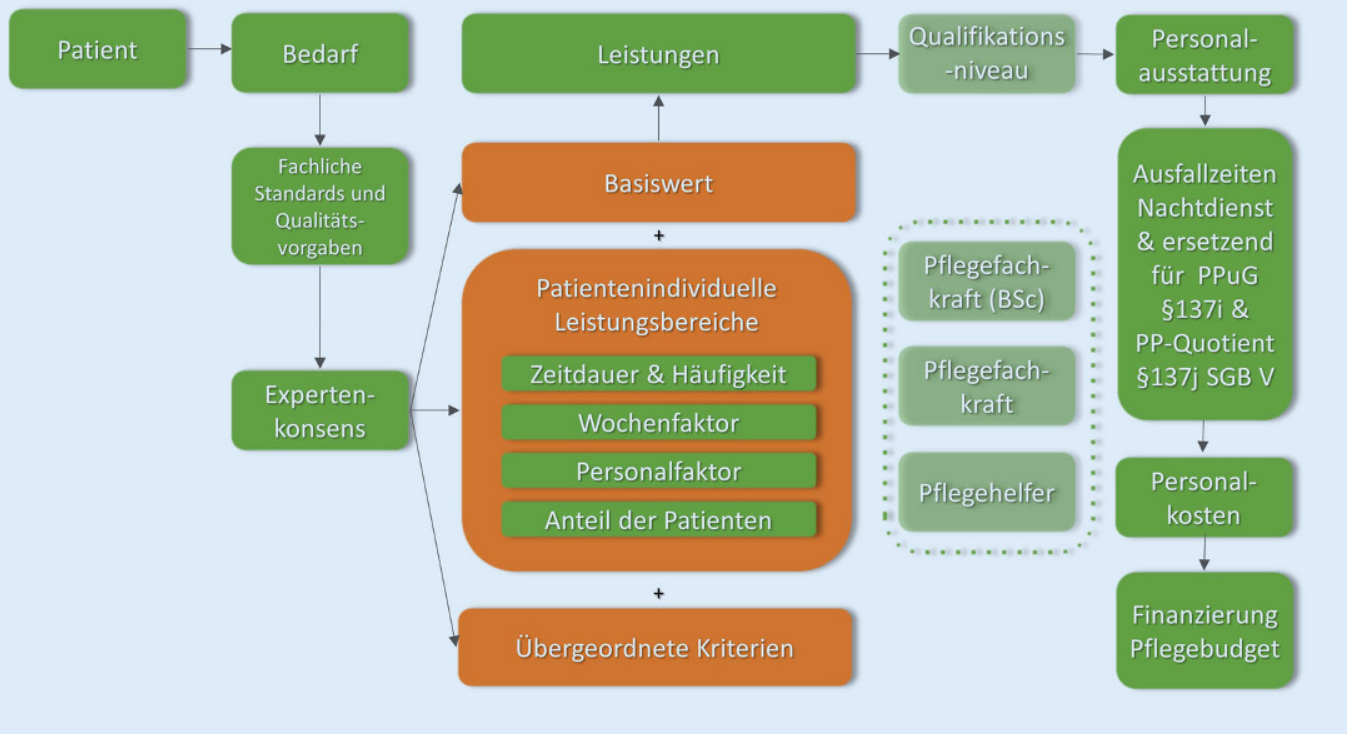

Abb. $2 \triangleleft$ Schematische Darstellung der Pflegepersonalregelung PPR 2.0. PPUG Pflegepersonaluntergrenzen, PP-Quotient Pflegepersonal-Quotient, beschreibt das Verhältnis der Anzahl der Vollzeitkräfte in der unmittelbaren Patientenversorgung auf bettenführenden Stationen eines Krankenhauses im Verhältnis zum Pflegeaufwand $S G B V$ Fünftes Buch Sozialgesetzbuch de vom Institut für Pflege- und Gesundheitswissenschaft der Medizinischen Fakultät, Martin-Luther-Universität HalleWittenberg durchgeführt.

Die teilnehmenden Einrichtungen wurden aufgefordert, Daten und Einschätzungen über einen zusammenhängenden Zeitraum von 7 Tagen zu liefern. Es wurden dabei PPR (alt) und PPR 2.0 parallel kodiert. Zur Anwendung wurde ein Handout mit den Einstufungsregeln für die Patienten mitgeliefert. Es erfolgten keine weiteren Anwenderschulungen. In die Untersuchung sind dabei Daten aus 206 Stationen und 25 Fachbereichen für insgesamt 31.347 Patiententage eingegangen.

Hinsichtlich Anwendbarkeit und Nutzbarkeit gab es 90 Rückmeldungen der Anwender. Diese ziehen ein positives Fazit. Die Einordnung im A-Bereich der PPR 2.0 empfanden $71 \%$ der Anwender als leicht, für den S-Bereich waren es $46 \%$. Keine Erleichterung war es für den A-Bereich lediglich für $13 \%$ und für den S-Bereich lediglich für $21 \%$. Betrachtet man dabei, dass der Anwendung keine Schulungen vorausgegangen sind und die Stufen A4 und S4 vollkommen neu konstruiert wurden, sind beide Werte überaus gut. Das Handout zur Anwendung der PPR 2.0 war für $60 \%$ eine große Hilfe bei der Einstufung, lediglich $13 \%$ verneinten dies.

Zum Verhältnis zwischen PPR (alt) und PPR 2.0 zeigt die 0 Tab. 1 deutlich, dass die PPR 2.0 in gleichem Maße fähig ist, die Patienten in den Aufwandsgruppen zu unterscheiden. Die geringfügigen und aufwandsgruppengerechten Verschiebungen sind bedingt durch die Einführung einer von 4 neuen Aufwandsgruppen im Zusammenhang mit der Bildung einer neuen S4-Stufe. In diese fallen $1,4 \%$ der eingeschätzten Patienten. Im Vergleich A4 alt vs. 2.0 gibt es ebenfalls eine sehr gute Entsprechung von $4,4 \%$ vs. $6,8 \%$.

\section{Voraussetzungen zur Umsetzung}

Um die PPR 2.0 erfolgreich und nachhaltig in den Alltag der Krankenhäuser zu implementieren und eine bedarfsgerechte Patientenversorgung zu gewährleisten, sind die erforderlichen Rahmenbedingungen zu schaffen. Hierzu haben DPR, ver.di und DKG in einem Eckpunktepapier Anforderungen an die Umsetzung formuliert [14].

\section{》) Unabdingbar ist die vollständige Refinanzierung der Pflege für die unmittelbare Patientenversorgung}

Unabdingbare Grundvoraussetzung für die nachfolgenden Punkte ist die vollständige Refinanzierung der Pflegepersonalkosten für die unmittelbare Patien- tenversorgung auf bettenführenden Stationen auf der Grundlage der PPR 2.0. Die gesetzlichen Vorgaben zu Pflegepersonaluntergrenzen in $\$ 137$ i SGB V sowie zum Pflegequotienten nach $\$ 137$ j SGB V erübrigen sich mit Einführung des neuen verbindlichen Instruments, denn die PPR 2.0 geht als bedarfsbezogenes Instrument über diese Vorgaben hinaus und beschränkt sich nicht auf „pflegesensitive" Bereiche.

\section{Pflegebudget und Stellenplan - Jahreszeitraum}

Die PPR 2.0 soll zukünftig die Grundlage für die Personalbemessung in den Pflegebudgetverhandlungen sein. Zusätzlich sind dabei in der Budgetvereinbarung u. a. Nachtdienste und Ausfallzeiten (soweit nicht in der PPR 2.0 berücksichtigt), einschließlich Stellen für Ausfallkonzepte (z. B. Pool) zu berücksichtigen. Maßstab für die Berechnung von Ausfallzeiten bilden grundsätzlich die Werte des Vorjahres.

\section{Grundsätze der Dienstplan- gestaltung- Dienstplanzeitraum}

Im Dienstplan einer Station/eines Arbeitsbereichs wird so viel Personal vorgesehen, wie anhand des gemessenen Pflegepersonalbedarfs nach PPR 2.0 des entsprechenden Vergleichszeitraums im Vorjahr benötigt wird. Erwartete oder bekannte Veränderungen gegenüber dem Vergleichszeitraum fließen dabei 
Tab. 1 Vergleichende Einordnung der

Pflegepersonalregelungen PPR (alt) und

PPR 2.0

\begin{tabular}{|l|l|l|}
\hline & PPR (\%) & PPR 2.0 (\%) \\
\hline A1/S1 & 15,0 & 16,2 \\
\hline A1/S2 & 17,1 & 17,0 \\
\hline A1/S3 & 3,5 & 3,9 \\
\hline A1/S4 & - & 0,1 \\
\hline A2/S1 & 7,8 & 8,7 \\
\hline A2/S2 & 23,5 & 21,4 \\
\hline A2/S3 & 7,9 & 7,3 \\
\hline A2/S4 & - & 0,5 \\
\hline A3/S1 & 3,2 & 3,7 \\
\hline A3/S2 & 12,7 & 10,2 \\
\hline A3/S3 & 4,9 & 3,6 \\
\hline A3/S4 & - & 0,5 \\
\hline A4/S1 & 0,7 & 0,8 \\
\hline A4/S2 & 2,5 & 4,1 \\
\hline A4/S3 & 1,2 & 1,6 \\
\hline A4/S4 & - & 0,3 \\
\hline
\end{tabular}

ebenfalls ein, soweit sie abschätzbar sind. Darüber hinaus sind die Vorgaben zur Nachtdienstbesetzung zu beachten. Bei absehbar drohender Unterschreitung der Regelbesetzung sind Maßnahmen zu deren Ausgleich zu veranlassen. Die Letztverantwortung für den Dienstplan liegt bei der Pflegedirektion/ Pflegedienstleitung.

\section{Ausfallkonzept}

Ziel des Ausfallkonzepts ist es, die im Dienstplan vorgesehene Besetzung verlässlich einzuhalten. Zum Ausgleich kurzfristiger Personalausfälle sind geeignete Ausfallkonzepte vorzuhalten. $\mathrm{Die}$ betrieblichen Interessensvertretungen sind auf Basis der geltenden Rechtsvorschriften $\mathrm{zu}$ beteiligen. Das Ausfallkonzept muss

- regeln, wann es aufgrund von Überlastung anzuwenden ist,

- dem Arbeits- und Gesundheitsschutz Rechnung tragen, indem Überlastung in kurzer Frist beseitigt wird,

- so viel Personal vorsehen, dass die im Krankenhaus gemessenen durchschnittlichen Ausfallzeiten kompensiert werden,

- geeignet sein, kurzfristige Dienstplanänderungen zu vermeiden, und
- Ausgleich der Arbeit unter Überlastung regeln.

\section{Personalausstattung für alle bettenführenden Stationen/ Arbeitsbereiche im Nachtdienst}

Auch im Nachtdienst ist eine bedarfsgerechte Patientenversorgung sicherzustellen. Sowohl die (alte) PPR als auch die PPR 2.0 sind keine Instrumente für den Nachtdienst. Die Besetzung im Nachtdienst soll daher weiterhin normativ geregelt werden. Es wird eine personelle Regelbesetzung für die Nachtdienste von grundsätzlich mindestens 2 Pflegepersonen, davon mindestens eine Pflegefachkraft, festgelegt. Bei mehr als 40-50 Patienten auf einer Station sind mindestens 2 Pflegefachkräfte vorzusehen. Für kleine Stationen bis zu 20 Patienten kann nach unten abgewichen werden (eine Pflegefachkraft); dabei muss eine Unterstützung in schwierigen Situationen und für Pausen vorgesehen werden. Für besonders pflegeaufwendige Bereiche sind darüber hinausgehende Besetzungen zu definieren.

\section{Transparenz}

Die Krankenhäuser dokumentieren die Sollbesetzung (VK) laut Dienstplan und die Istbesetzung jeder Station/jedes Arbeitsbereichs (VK) für jeden Dienstplanzeitraum (i.d. R. monatlich). Die betriebliche Interessenvertretung erhält Zugang zu diesen Daten. Der Soll-Ist-Vergleich auf der Fachabteilungsebene wird jährlich im Intranet des Krankenhauses veröffentlicht.

\section{Übergangsregelungen}

Zurvollständigen Umsetzung der PPR 2.0 werden flankierende Maßnahmen umgesetzt, u.a. Erhöhung der Zahl der Ausbildungsplätze in der Pflege, Rückgewinnung von aus dem Beruf Ausgeschiedenen und Bindung von Pflegekräften, Erhöhung der Stunden von Teilzeitbeschäftigten in der Pflege, zusätzliche Maßnahmen zur Vereinbarkeit von Familie und Beruf und weitere Maßnahmen im Sinne der KAP.

Mit Inkrafttreten der PPR 2.0 wird ein Umsetzungsgrad krankenhausindividuell festgestellt, und es beginnt der Pflegepersonalaufbau in jedem Kran- kenhaus. Der Umsetzungsgrad der sich aus der PPR 2.0 ergebenen Personalausstattung wird vom Inkrafttreten der PPR 2.0 an stufenweise erhöht. Dabei sollen sich die Stufen an ambitionierten, aber auch realisierbaren Werten orientieren und die Erfahrungen aus der PPR 2.0 und deren Umsetzung berücksichtigen. Die weiteren Schritte und Fristen zur Erreichung eines Umsetzungsgrads von $100 \%$ werden 2 Jahre nach Inkraftsetzung der PPR 2.0 und erfolgter Evaluation festgelegt. Bis $\mathrm{zu}$ diesem Zeitpunkt erfolgt eine jährliche Zusammenführung zur Ermittlung eines bundesdurchschnittlichen Umsetzungsgrads durch ein wissenschaftliches Institut.

\section{Intensivmedizin/Pädiatrie}

Für die Pflegepersonalbemessung der Intensivmedizin und der Pädiatrie soll in einem weiteren Schritt möglichst zeitnah ein Instrument entwickelt und umgesetzt werden. Dabei werden bestehende Vorgaben, Empfehlungen und Instrumente berücksichtigt.

\section{Ausblick}

Die PpUG erfüllen nicht die Erwartungen der Kolleginnen aus der Pflegepraxis und können nicht helfen, einen strukturierten und bedarfsorientierten Stellenaufbau einzuläuten. Mit der PPR 2.0 liegt jetzt ein unmittelbar einsatzfähiges Instrument zur Messung des Pflegepersonalbedarfs vor, das dem BMG am 13. Januar 2020 präsentiert wurde. Flankiert werden muss die PPR 2.0 durch weitere Maßnahmen aus der KAP, um die Attraktivität der Pflegeberufe zu steigern, damit sich mehr Menschen für die Arbeit in einem Pflegeberuf entscheiden.

\section{Fazit für die Praxis}

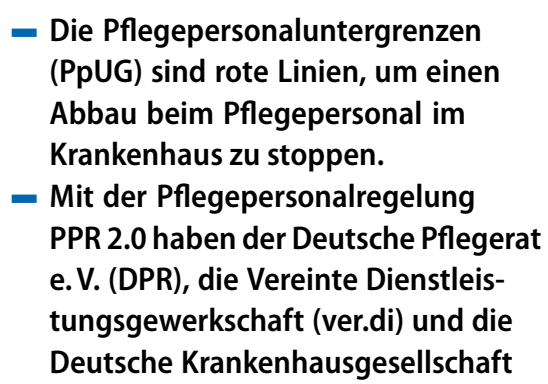


(DKG) entsprechend ihrer Selbstverpflichtung in der Konzertierten Aktion Pflege (KAP) gemeinsam eine Interimslösung zur Einführung der Pflegepersonalbemessung entwickelt und einem umfangreichen Pretest erfolgreich unterzogen.

- Das Bundesministerium für Gesundheit (BMG) ist nun aufgefordert, rasch die zur Einführung der PPR 2.0 notwendigen gesetzlichen Regelungen vorzugeben und parallel dazu den zweiten Schritt einer notwendigen wissenschaftlichen (Weiter-)Entwicklung in Gang zu setzen.

\section{Korrespondenzadresse}

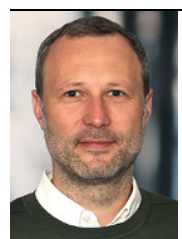

Prof. Dr. P. Jahn

Department für Innere Medizin, AG Versorgungsforschung, Pflege im Krankenhaus, Universitätsmedizin Halle (Saale) Ernst-Grube-Straße 40, 06120 Halle (Saale), Deutschland patrick.jahn@uk-halle.de

\section{Einhaltung ethischer Richtlinien}

Interessenkonflikt. P. Jahn gibt an, dass kein Interessenkonflikt besteht.

Für diesen Beitrag wurden von den Autoren keine Studien an Menschen oder Tieren durchgeführt. Für die aufgeführten Studien gelten die jeweils dort angegebenen ethischen Richtlinien.

\section{Literatur}

1. Westerfellhaus A (2020) Positionspapier „Mehr PflegeKRAFT 2.0" - Pflege ist mehr als systemrelevant. https://www.pflegebevollmaechtigter.de/ details/mehr-pflegekraft-2-0.html. Zugegriffen: 28. Mai 2020

2. Leopoldina (2020) 4. Ad-hoc-Stellungnahme Coronavirus-Pandemie: Medizinische Versorgung und patientennahe Forschung in einem adaptiven Gesundheitssystem. https://www.leopoldina. org/uploads/tx_leopublication/2020_05_27_ Stellungnahme_Corona_Gesundheitssystem.pdf. Zugegriffen: 28. Mai 2020

3. Simon M (2018) Von der Unterbesetzung in der Krankenhauspflege zur bedarfsgerechten Personalausstattung | Eine kritische Analyse der aktuellen Reformpläne für die Personalbesetzung im Pflegedienst der Krankenhäuser und Vorstellung zweier Alternativmodelle. https:// www.boeckler.de/pdf/p_fofoe_WP_096_2018. pdf. Zugegriffen: 28. Mai 2020 (Working Paper For- schungsförderung Hans Böckler Stiftung, Nummer 096, Oktober 2018)

4. In E (2019) Handbuch zur Kalkulation von Behandlungskosten Version 4.0 10. Oktober 2016. https://www.g-drg.de/ Kalkulation2/DRG-Fallpauschalen_17b_KHG/ Kalkulationshandbuch. Zugegriffen: 28. Mai 2020

5. Schreyögg J, Milstein R (2016) Expertise zur Ermittlung des Zusammenhangs zwischen Pflegeverhältniszahlen und pflegesensitiven Ergebnisparametern in Deutschland. https://www. bundesgesundheitsministerium.de/fileadmin/ Dateien/5_Publikationen/Pflege/Berichte/ Gutachten_Schreyoegg_Pflegesensitive_ Fachabteilungen.pdf. Zugegriffen: 28. Mai 2020

6. Needleman J et al (2002) Nurse-staffing levels and the quality of care in hospitals. N Engl J Med 346(22):1715-1722

7. Aiken L et al (2010) Implications of the California nurse staffing mandate for other states. Health Serv Res 45(4):904-921

8. Augurzky B, Bünnings $C$, Dördelmann S, Greiner W, Hein L, Scholz S, Wübker A (2016) Die Zukunft der Pflege im Krankenhaus, Heft 104. https://www.rwi-essen.de/media/ content/pages/publikationen/rwi-materialien/ rwi-materialien_104.pdf. Zugegriffen: 28. Mai 2020

9. IGES (2017) Faktencheck - Pflegepersonal im Krankenhaus | Internationale Empirie und Status quo in Deutschland, Bertelsmann Stiftung. https:// faktencheck-gesundheit.de/de/publikationen/ publikation/did/faktencheck-pflegepersonal-imkrankenhaus/.Zugegriffen:28. Mai 2020

10. DBfK (2020) Ziel erreicht? Ergebnisse einer OnlineUmfrage zu Effekten der Pflegepersonaluntergrenzen im Krankenhaus. https://www.dbfk. de/media/docs/download/Allgemein/BerichtUmfrage-Pflegepersonaluntergrenzen-2020-0519.pdf. Zugegriffen: 28. Mai 2020

11. Thomas D, Reifferscheid A, Pomorin N, Wasem J (2014) Instrumente zur Personalbemessung und -finanzierung in der Krankenhauspflege in Deutschland IBES Diskussionsbeitrag, Nr. 204, September 2014. https://gesundheit-soziales. verdi.de/++file++5832c9aef1b4cd68fea7792e/ download/DP\%20204-Wasem-endg.pdf. Zugegriffen:28. Mai 2020

12. Konzertierte Aktion Pflege (2019) Vereinbarungen der Arbeitsgruppen 1 bis 5. https://www. bundesgesundheitsministerium.de/fileadmin/ Dateien/3_Downloads/K/Konzertierte_Aktion_ Pflege/191129_KAP_Gesamttext_Stand_11. 2019_3._Auflage.pdf.Zugegriffen:28. Mai 2020

13. Material zur PPR 2.0 https://www.dkgev.de/ themen/personal-weiterbildung/ppr-20/. Zugegriffen: 8. Mai 2020

14. Eckpunkte zur Umsetzung der PPR 2.0. https://gesundheit-soziales.verdi. de/++file++5e1dbaa8322ec4d3fa916945/ download/20200113_Eckpunkte_Umsetzung_ PPR2.0.pdf.Zugegriffen:28. Mai 2020
Hier steht eine Anzeige. 黛 Springer 
Hier steht eine Anzeige.

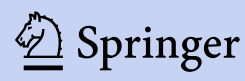

